\title{
STABLE REPETITIVE CONTROL BY FREQUENCY ALIASING
}

\author{
James D. Ratcliffe, Paul L. Lewin and Eric Rogers \\ University of Southampton \\ School of Electronics and Computer Science, University Road, Southampton, SO17 1BJ, United Kingdom \\ Email: jdr@ecs.soton.ac.uk \\ Jari J. Hätönen, Thomas J. Harte and David H. Owens \\ University of Sheffield \\ Department of Automatic Control and Systems Engineering, Mappin Street, Sheffield, S1 3JD, United Kingdom \\ Email: J.Hatonen@sheffield.ac.uk
}

\begin{abstract}
Keywords: Repetitive control, signal processing.
Abstract: A filtering technique based on frequency aliasing which was initially developed for Iterative Learning Control is modified so that it can be implemented in real-time and is suitable for Repetitive Control. The aliasing technique is experimentally verified on a gantry robot facility, which manipulates payloads from a dispenser onto a constant velocity conveyor. A parallel arrangement consisting of a three-term feedback controller and a simple structure repetitive controller is used to control the robot. Without the aliasing technique, the combined control system becomes unstable very rapidly. In contrast, when the aliasing technique is applied, 1000 repetitions are successfully completed and no indications of impending instability can be seen.
\end{abstract}

\section{INTRODUCTION}

Repetitive Control (RC) is concerned with improving the performance of trajectory tracking and setpoint control problems in the cases where the reference trajectory is repeated ad infinitum or the disturbances are repeated and occur in a regularly timed manner. Examples of this type of application include food production facilities, printing presses and bulk product transfer systems such as conveyors. Applications such as these, tend to be controlled by standard feedback techniques such as the three-term or PID controller. This is because they are cheap, robust methods which are relatively simple to install and tune, and the production facility can be made operational rapidly.

The disadvantage of using a simple structure feedback controller is that for a repetitive process, the same level of error will be obtained for each repetition. It is also likely that as the plant dynamics change, due to wear for example, the feedback controller will gradually become de-tuned and control performance will reduce, necessitating regular retuning to counteract this.

Repetitive control utilises the error data from previous executions of the task, to modify the input profile, which is supplied to the plant at the next trial, in a way which seeks to reduce the tracking error. Theoretically, this technique will converge asymptotically to zero tracking error, if non-repeating disturbances are not present. This is a property which should make $\mathrm{RC}$ very attractive to industrial applications. However, RC also has some disadvantages particularly concerned with algorithm stability. Generally, an algorithm is found to reduce the tracking error for a number of iterations, but then gradually becomes unstable and the tracking error increases exponentially. Evidently, this type of behaviour is unacceptable in an industrial context.

It has generally been recognised that this unstable behaviour is caused by certain signals, which can build up within the cyclic learning loop. In particular, these signals include high frequency noise, or low frequency signals which have an input/output phase shift larger than $180^{\circ}$. Simple structure learning techniques are unable to compensate for these signals (Chen and Longman, 2002). Therefore at each repetition, more are added to the learnt signal until this becomes corrupted to the point where the plant cannot operate. Standard practice to prevent instability from occurring is to filter these signals from the error data. Unfortunately, conventional filtering techniques induce additional phase lag into the control system, which results in reduced controller performance and non-zero residual tracking error. It is therefore necessary to filter the data without introducing additional phase shift, by using zero-phase methods.

This paper investigates the development of stable, 
simple structure, repetitive control techniques by using a frequency aliasing method, as a means of filtering unwanted information from the learning loop and hence maintaining stability, in an inherently unstable control system. The aliasing technique was originally developed by the authors in the ILC context (Ratcliffe et al., 2005a), but can be ported to the RC context, to provide a simple, real-time zero-phase filtering technique. In contrast, many stabilisation techniques in $\mathrm{RC}$ rely upon the use of batch zero-phase filtering, which is not easily implemented in real-time, as it is inherently a non-causal technique. Achieving batch zero-phase filtering in RC systems, involves operating the plant and simultaneously batch filtering data over a period of several repetitions, see for example (Chen and Longman, 1999; Songchon and Longman, 2001).

\section{REAL-TIME ALIASING}

Shannon's sampling theorem states that to record a signal of a given frequency in a discrete-time system, the sample frequency must be at least twice the frequency of the signal. If this requirement is not met, then frequency aliasing occurs and the higher frequency components of the original signal are lost.

In most applications, aliasing is a drawback, usually redressed by means of anti-aliasing filters. In this application, aliasing is beneficially used to remove the effects of the undesired frequencies from the learning controller. The controller configuration uses a simple structure P-type repetitive controller in parallel with a PID feedback controller. The feedback controller is required to smooth the output produced by the aliasing technique, but is also useful for producing good tracking performance during the first few repetitions. The feedback controller also provides additional robustness to non-repeating disturbances and can respond to sudden disturbances much more rapidly than the learning controller. In addition to the feedback and feed-forward controllers, an additional aliasing module is implemented on the output generated by the learning controller. The aliasing module samples the output generated by the learning controller at a frequency less than twice the lowest frequency which needs to be removed from the learning process. In this way, undesired frequencies are aliased to a lower frequency, while the aliasing filter still allows the RC to learn frequencies below the alias cut-off. As long as the cut off frequency is greater than the frequencies which need to be learnt to satisfy the reference demand, the loss of data caused by aliasing does not prevent the learning controller from improving the performance of the overall system.

In the following analysis, 'alias frequency' is used to describe the sample frequency of the aliasing filter. 'Feedback frequency' is used to describe the sample frequency of the remaining control system $(1 \mathrm{kHz})$. 'Alias gap' $(a g)$ is used to relate the alias frequency to the feedback frequency. The alias gap is the number of feedback frequency sample instants between each alias frequency sample instant.

Using a zero-order-hold approach for the aliasing module is not suitable, as the aliasing filter generates a signal, which relates poorly to the original nonaliased signal, due to large step changes in the control voltage. In a non-causal approach which can be used for ILC, instead of holding the signal value constant between aliasing samples, it is possible to calculate the gradient between adjacent samples and join them by linear interpolation, a technique which is commonly used in practical signal processing. Following the successful completion of an iteration, the ILC algorithm computes the next ILC component of the plant input vector at feedback frequency. This input vector is likely to contain unwanted frequencies. The aliasing module then re-samples the input vector at the aliasing frequency, removing all frequencies above the aliasing cut-off. The input vector now consists of far fewer sample instants than are required for real-time operation. Linear interpolation is used to connect the aliasing frequency sample instants, so that a smooth signal is produced when the interpolated signal is re-sampled at feedback frequency. During the next iteration, each sample instant of the aliased ILC vector is summed with the input produced by the feedback controller.

The same principle can be implemented in repetitive control in a causal manner, by delaying the learning update by a number of samples equal to the alias gap. The linear interpolation method can then be performed one sample at a time, rather than in a batch. The flowchart in Figure 1 describes the sequence of events.

\section{TEST FACILITY}

The experimental results presented in Sections 4 and 5 were obtained by implementing the parallel configuration controller on the test facility depicted in Figure 2. It consists of a 3-axis gantry robot mounted above one end of a plastic chain conveyor. The robot is required to collect payloads from an asynchronous dispenser, then transfer them to the conveyor which is travelling at constant velocity. Once the payloads reach the opposite end of the conveyor, they are fed into a return mechanism which cycles the payloads back to the dispenser. This allows many repetitions to be performed, with a minimal number of payloads. The robot and conveyor emulate many synchronous 


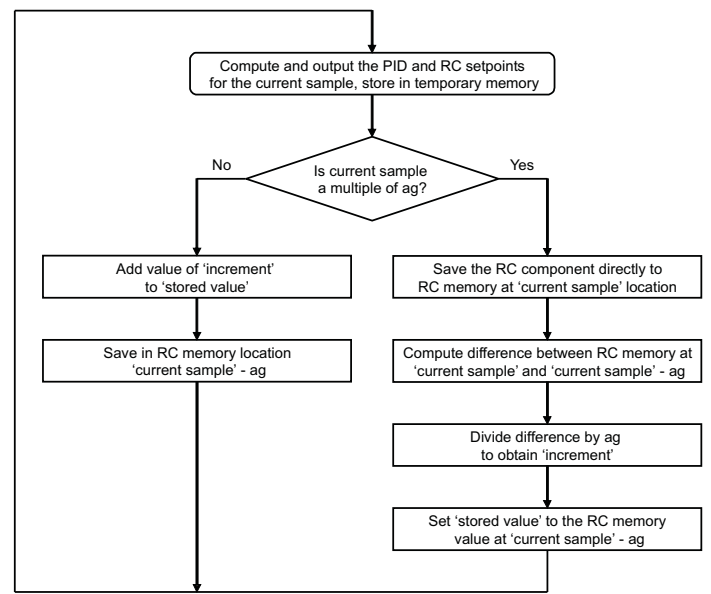

Figure 1: Program operation flowchart $(a g=$ alias gap $)$

placement operations which can be found in industry. A detailed description of the robot and models for each axis can be found in (Ratcliffe et al., 2005b).

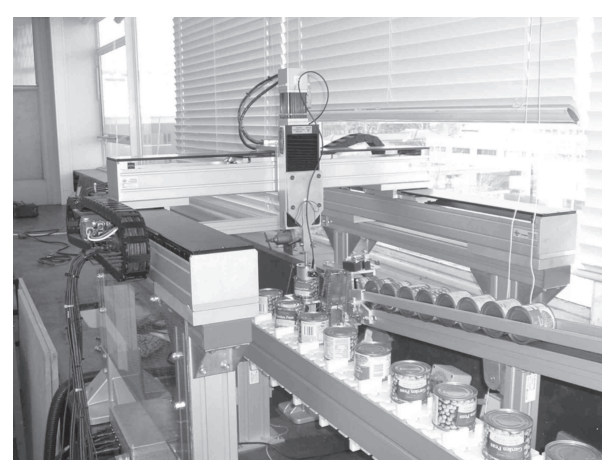

Figure 2: Gantry robot, conveyor and payload dispenser

\section{INITIAL IMPLEMENTATION}

Before implementing the aliasing technique, the plant is operated without any form of filtering. The feedback controller and the learning controller both operate with a sample frequency of $1 \mathrm{kHz}$. Figure 3 displays the mse results for each axis on a logarithmic vertical scale. The learning gain $\beta$ was set to 10 and 100. The values of mse for iteration 1 represent the tracking performance, which is achieved by the feedback controller alone, because at this stage the repetitive controller has had no opportunity to learn anything from previous trials. Clearly, the simple structure repetitive controller can significantly improve the tracking performance, as the mse for each axis is reduced by approximately two orders of magnitude.
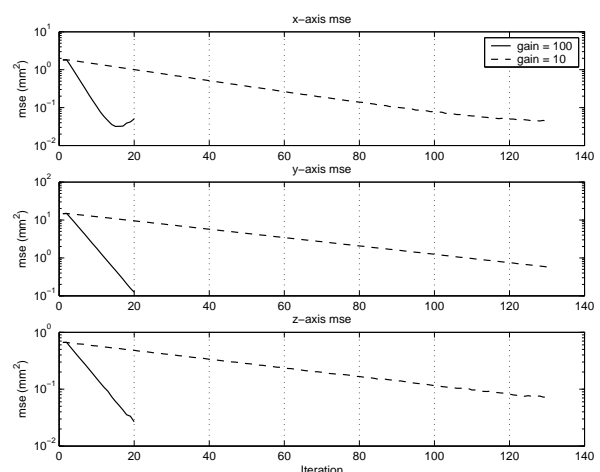

Figure 3: mse for 1000 repetitions with alias gap $=70$

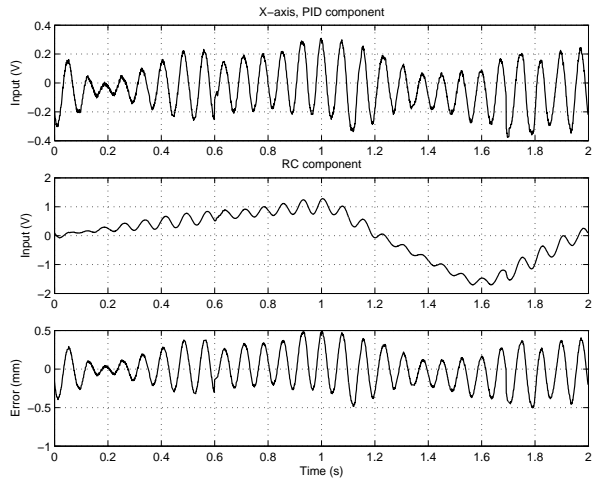

Figure 4: $X$-axis inputs and tracking error (repetition 20)

Higher learning gain also results in faster convergence to minimum error as expected. However, with $\beta$ equal to 100 , the $X$-axis mse begins to increase after 17 iterations, which is a clear indication of instability. This is less noticeable for $\beta$ equal to 10 , but for both values of gain, the robot visibly began to shake as the algorithm became unstable.

Figure 4 shows the $X$-axis feedback controller input, repetitive controller input and measured tracking error for iteration 20 with $\beta$ equal to 100 . All three signals are heavily corrupted by a sinusoid of frequency $13-14 \mathrm{~Hz}(81-87 \mathrm{rad} / \mathrm{s})$. Referring to frequency response plots for the robot axes, this frequency corresponds well to a pair of complex conjugate poles in the dynamic response of the $X$-axis. Effectively, a resonant frequency is allowed to build up within the learning loop.

\section{WITH ALIASING}

To restore stability, it is necessary to prevent this resonant frequency from building up in the learning loop. With the system operating at a sample frequency of 1 

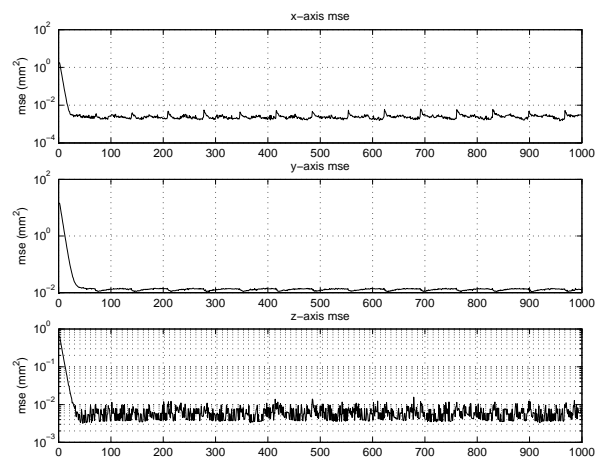

Figure 5: mse for 1000 repetitions (alias gap $=70, \beta=100$ )

$\mathrm{kHz}$, an alias gap of 70 produces an aliasing frequency of $14.28 \mathrm{~Hz}$. Shannon's sampling theorem then stipulates that only signals of half this frequency can be recorded. Therefore, the aliasing module removes all signals of frequency larger that $7.14 \mathrm{~Hz}$, including the resonant frequency between 13 and $14 \mathrm{~Hz}$. Figure 5 displays the mse results for 1000 repetitions, when the aliasing module is implemented with a alias gap of 70 and the learning gain is equal to 100 . The mse is reduced by over two orders of magnitude for each axis and although it oscillates about a mean value, there is no indication that it increases. The 1000 repetition test does not guarantee that the control system will be stable for an infinite number of repetitions. However, compared to the original controller without aliasing which achieved 20 repetitions, there is a significant improvement.

Figure 6 shows the $X$-axis controller inputs and recorded tracking error for iteration 1000 with the alias gap equal to 70 . Although the tracking error still contains sinusoidal oscillations in the frequency range $13-14 \mathrm{~Hz}$, these are clearly being stimulated by the feedback controller and not the learning controller. As the feedback controller has no learning or memory capabilities, the resonant frequency cannot grow at each repetition.

Referring back to Figure 5, the underlying cause behind the cyclic variation in mse is not yet understood and requires further investigation. One possible reason which can be suggested immediately is that the variation is related to the alias gap number. To investigate this hypothesis, the 1000 repetition test was performed with an alias gap of 100 . The increase in alias gap value has a direct influence on the error reduction, because the frequency range which can pass through the aliasing module has been reduced. Therefore the error reduction is now less than two orders of magnitude. However, the amplitude of the cyclic variation in mse has been greatly reduced and the cycles now occur every 50 repetitions. Evidently there is a link between the alias gap and the variation which requires further investigation.

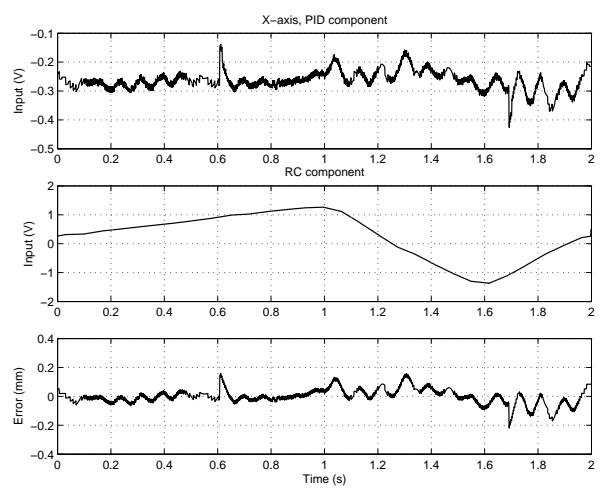

Figure 6: $X$-axis inputs and tracking error (repetition 1000 , $a g=70, \beta=100)$

\section{CONCLUSIONS}

The aliasing technique which was initially developed for iterative learning control has been successfully converted to a simple, causal operation which can be calculated at each sampling instant in a repetitive control context. Experimental validation demonstrates that the stability of a simple structure repetitive controller can be greatly improved by using the aliasing technique.

\section{REFERENCES}

Chen, H.-J. and Longman, R. (1999). The importance of smooth updates in producing good error levels in repetitive control. In Proceedings of the 38th IEEE Conference on Decision and Control, Phoenix, Arizona, pages 258-263.

Chen, K. and Longman, R. (2002). Stability issues using fir filtering in repetitive control. Advances in the Astronautical Sciences, 112(2):1321-1340.

Ratcliffe, J., Hätönen, J., Lewin, P., Rogers, E., Harte, T., and Owens, D. (2005a). P-type iterative learning control for systems that contain resonance. Accepted by International Journal of Adaptive Control and Signal Processing.

Ratcliffe, J., van Duinkerken, L., Lewin, P., Rogers, E., Hätönen, J., Harte, T., and Owens, D. (2005b). Fast norm-optimal iterative learning control for industrial applications. In Proceedings of the 24th American Control Conference, Portland, pages 1951-1956.

Songchon, T. and Longman, R. (2001). On the waterbed effect in repetitive control using zero-phase filtering. Advances in the Astronautical Sciences, 108:13211340. 